\title{
Context-Aware Cloud Computing for Construction Collaboration
}

\author{
Mohamad Syazli Fathi ${ }^{1}$, Mohammad Abedi ${ }^{2}$, Shuib Rambat ${ }^{3}$, \\ Shakila Rawai ${ }^{2}$ and Mohd Zulakhmar Zakiyudin ${ }^{4}$ \\ ${ }^{1}$ Department of Civil Engineering, UTM Razak School of Engineering \& Advanced Technology, Universiti \\ Teknologi Malaysia, Kuala Lumpur, Malaysia \\ ${ }^{2}$ Faculty of Civil Engineering, Universiti Teknologi Malaysia, Johor, Malaysia \\ ${ }^{3}$ School of Professional and Continuing Studies, Universiti Teknologi Malaysia, Jalan Semarak, \\ Kuala Lumpur, Malaysia \\ ${ }^{4}$ Faculty of Architecture and Built Environment, Twintech International University College of Technology, \\ Kuala Lumpur, Malaysia
}

\begin{abstract}
The construction industry has been characterised by fragmentation, lack of integration and complexities in processes and activities. These characteristics could threaten the delivery of project objectives which could ultimately have a negative impact on construction project success. The construction industry is associated with numerous activities, processes, organisations and individuals. Improving the collaboration between the parties within the construction industry will enhance productivity whilst maximising efficiency and effectiveness. Providing the construction industry parties with access to accurate and up-to-date information along with efficient communications via a reliable, competent and appropriate network is frequently a challenge. The main objective of this paper is to investigate the construction collaboration tools along with the concepts of cloud computing and context-awareness. The findings in this research are based on a thorough review of the comprehensive literature on IT, computing and construction. Accordingly, this study presents and develops the concepts and potential of innovative collaborative tools, such as Context-Aware Cloud Computing Information Systems (CACCIS). Implementing CACCIS in the construction industry will facilitate the construction supply chain processes, relations and networks along with increasing the opportunities for enhancing competitive advantages. Firstly, it is hoped that this study will contribute to improvements in construction industry collaboration which could ultimately improve and enhance its competitive advantages. Secondly, it proposes a fresh vision of construction industry integration and, most significantly, collaboration in an efficient way that will eventually maximize productivity and enhance the efficiency and effectiveness of the construction industry.
\end{abstract}

Keywords: Construction Industry, Construction Collaboration Tools, Cloud Computing, ContextAwareness.

Copyright (C) 2012 Mohamad Syazli Fathi, Mohammad Abedi, Shuib Rambat, Shakila Rawai and Mohd Zulakhmar Zakiyudin. This is an open access article distributed under the Creative Commons Attribution License unported 3.0, which permits unrestricted use, distribution, and reproduction in any medium, provided that original work is properly cited. Contact author: Mohamad Syazli Fathi E-mail: syazli@utm.my 


\section{Introduction}

The construction industry is often associated with fragmentation, complexities and nonintegrated environments. The significant challenge for construction projects is to integrate, support and facilitate a multidisciplinary team of stakeholders working with supply chain parties and project stakeholders on achieving the common goals of a project. They must work collaboratively to improve the productivity and effectiveness of construction management and to ensure the efficient utilisation of resources in order to guarantee the success of construction projects in achieving their specific objectives. These specific objectives mostly concern completing the project within the required time and cost whilst ensuring its quality and safety. For several years, the construction industry has made considerable efforts within an increasingly competitive market to implement techniques, technologies and tools that can reduce project duration and cost along with improving productivity, efficiency and effectiveness. It should be noted that clients within the dynamic construction industry are requesting better value of money, higher quality, shorter construction cycle times and access to up-todate information. Moreover, they require such information to be produced at any point in both the project life cycle and construction supply chain (O'Brien et al., 2011; Aziz et al., 2006).

Although a variety of technological advancements have arisen within the construction industry (Anumba et al., 2008 and 2006; Sze-wing et al., 2008; Acar et al., 2005), there are still many challenges in existing construction processes resulting from poor access to the right information at the right time for effective decision-making and from a general communication breakdown between project participants. This could contribute to a lack of collaboration and integration both within the construction supply chain and between project stakeholders (Xuan et al., 2007;
Peansupap et al., 2006; Kondratova et al., 2003).

Quality, quantity and timing of information are significant elements in construction projects which can either hamper or assist the successful consequences for project objectives (Garza et al., 1998). Moreover, according to Anumba et al. (2008), construction is an information-intensive industry since information delivery is the key to better management within the construction supply chain including the implementation of construction projects. Also, it is vital for cost savings, efficient and effective decision making and a key to success. Modern project management approaches and tools have been evolved in order to overcome problems in construction management. The use of project management tools in construction management has been widely known (Milosevic, 2003). All of these techniques and tools are key facilitators for efficient and effective collaboration among construction industry parties.

In order to overcome the mobile and information-intensive nature of construction projects, their unstructured and dynamic nature, along with the difficulties and hazards existing within the construction industry, will necessitate the use of intelligent solutions such as adapting cloud computing to support the construction industry. As stated by Goscinski and Brock (2010), cloud computing is a new business model wrapped around new technologies, such as virtualisation, applications (Software as a Service (SaaS)), platforms (Platform as a Service (PaaS)) and hardware (Infrastructure as a Service (IaaS)). Therefore, this research effort is to discover the concepts and benefits of applying and incorporating a contextaware approach to the use of cloud computing within the construction industry.

Firstly, this paper presents how collaboration technologies can assist the professional parties in managing construction projects more effectively. It also presents the various definitions, delivery models and types of 
cloud computing. The third part of this research demonstrates the enabling technologies as Context-Aware Construction Collaboration Tools. The potential of ContextAware Cloud Computing Information Systems (CACCIS) as a proposed collaboration tool will be illustrated in part four of this research. Finally, the conclusion develops ways to enhance the potential opportunities offered to the construction industry by collaborative technologies and tools.

\section{Construction Collaboration Tools}

Since the early 2000's, many researchers have sought to implement techniques, technologies and tools which reduce project duration and cost whilst improving quality, productivity, efficiency and effectiveness. Within the dynamic construction industry, clients, as a significant part of the construction supply chain, are requesting better value for money, higher-quality products, shorter construction periods and access to valid and up-to-date information at any time in the project life cycle $\left(O^{\prime}\right.$ Brien et al., 2011). As asserted by Barthelmess (2003), collaboration in a project requires those involved to communicate and be aware of one another's activities. Moreover, collaboration requires successful and efficient sharing of knowledge, negotiation, coordination and management of activities
(Lang et al., 2002). On the other hand, the unstructured, dynamic and complicated nature of projects, their difficulties, threats and risks, are associated with the construction industry. Thus, in order to overcome the mobile and informationintensive nature of construction projects, the use of creative and intelligent ways of collaboration will be necessary. Consequently, cloud computing is a potentially significant tool to support the construction industry and its currently available collaboration tools, such as desktop PCs, internet networks, tablets, smartphones and laptops. Therefore, this research will propose the development of a context-aware construction collaboration tool (Application) for the successful effective implementation of cloud computing in the construction industry that could be utilized by the supply chain parties and project stakeholders. The next part of this research will present the various concepts, definitions, delivery models and types of cloud computing.

\section{Cloud Computing}

Cloud computing is the latest technology being developed by the IT industry as the next revolution that will influence how efficiently and effectively the internet and information systems could operate and be used worldwide. Table 1 illustrates the various definitions of cloud computing. 
Table 1: Definitions of Cloud Computing

\begin{tabular}{|l|l|}
\hline \multicolumn{1}{|c|}{ Author(s) } & \multicolumn{1}{c|}{ Definition } \\
\hline Liu et al. (2012) & Cloud computing has vast storage and powerful computing ability. \\
\hline Sultan et al. (2012) & $\begin{array}{l}\text { Clusters of distributed computers (largely vast data centres and server } \\
\text { farms), which provide on-demand resources and services over a } \\
\text { networked medium (usually the Internet). }\end{array}$ \\
\hline $\begin{array}{l}\text { Voorsluys } \text { et al. } \\
\text { (2011) }\end{array}$ & $\begin{array}{l}\text { A new computing paradigm which offers a huge amount of computational } \\
\text { and storage resources to the masses. }\end{array}$ \\
\hline $\begin{array}{l}\text { Mell and Grance } \\
\text { (2011) and Cloud } \\
\text { Computing (2010) }\end{array}$ & $\begin{array}{l}\text { Cloud computing is a model for enabling ubiquitous, convenient, on- } \\
\text { demand network access to a shared pool of configurable computing } \\
\text { resources. }\end{array}$ \\
\hline $\begin{array}{l}\text { Sultan (2010) } \\
\text { Goscinski and Brock } \\
\text { (2010) }\end{array}$ & $\begin{array}{l}\text { The IT capabilities that are requested, provisioned, delivered and } \\
\text { consumed in real time over the internet. } \\
\text { virtualisation, applications (Software as a Service (SaaS)), platforms } \\
\text { (Platform as a Service (PaaS)) and hardware (Infrastructure as a Service } \\
\text { (IaaS)). }\end{array}$ \\
\hline $\begin{array}{l}\text { Sharif (2010) } \\
\text { As a technology that has the potential of changing how the internet and } \\
\text { the information systems are presently operated and used. }\end{array}$ \\
\hline $\begin{array}{l}\text { Multan (2010); } \\
\text { Erdogmus (2009) and } \\
\text { Bittman, T.J. (2009) }\end{array}$ & $\begin{array}{l}\text { A collection of disembodied services accessible from anywhere using any } \\
\text { mobile device with an internet-based connection. }\end{array}$ \\
\hline $\begin{array}{l}\text { Armbrust } \text { et al. } \\
\text { (2009) }\end{array}$ & $\begin{array}{l}\text { These services are provided through applications delivered as a service } \\
\text { over the internet, the hardware and systems software in data-centres. }\end{array}$ \\
\hline $\begin{array}{l}\text { Erdogmus (2009) } \\
\text { A pool of highly scalable, abstracted infrastructure which is capable of } \\
\text { hosting end-customer applications that are billed according to } \\
\text { consumption. }\end{array}$ \\
\hline
\end{tabular}

Analysis from Table 1 shows that cloud computing can be defined as the sharing and utilization of applications and resources within a network environment to implement the business without any concerns regarding the ownership, management and maintenance of the network's resources, applications and services. Furthermore, according to Zhang et al. (2011), applications on smartphones are traditionally constrained by limited resources, such as low CPU frequency, small memory and a batterypowered computing environment. Therefore, cloud computing technology could be a significant alternative for solving these issues. It should be noted that this technology could be applied to overcome the issues and constraints asserted by Zhang et al. (2011) via smartphones and tablets etc.
There have been four delivery models, (deployment models) of cloud computing identified: public cloud, private cloud, community cloud and hybrid clouds. Armburst et al. (2009) defined a public cloud as when it is constructed in a pay-as-you-go manner to the public (open system for any users) and a private cloud when the internal data-centres of a business are not made available to the public (a closed system with access only for special users). The two other delivery models are community and hybrid. The community cloud is related to a cloud that is controlled and used by a group of organisations that have shared interests whilst the hybrid cloud is a combination of a public and private cloud that interoperates (Mell and Grance, 2011; Cloud Computing, 2010). 
According to Mell and Grance (2011); P.Y. Thomas (2011); Cloud Computing (2010); Reeves (2009); Kim (2009); Hoover and Martin (2008) and Foley (2008), there are three broad types of cloud computing, defined as follows:

(1) Infrastructure as a Service (IaaS): The consumer via the cloud providers uses fundamental computing resources such as processing power, storage, operating system, networking components such as firewalls or middleware. Amazon web services are a good instance in this category;

(2) Platform as a Service (PaaS): The consumer uses a hosting environment for their applications. They control the applications that run in the environment (possibly with some control over the hosting environment), but with no control over the operating system, hardware or network infrastructure on which they are running. Google AppEngine, Amazon's S3, EC2 offerings and Microsoft Azure are good examples of this category, and

(3) Software as a Service (SaaS): The consumer uses an application, but does not control the operating system, hardware or network infrastructure on which it is running. On the other hand, in the utilisation of SaaS, a provider licenses an application to customers as a service on demand, through a subscription, in a "pay-as-you-go" model. Examples of this category includes Zoho Office, Microsoft WindowsLive, a variety of Google Apps including Gmail, Dropbox, Google Talk, Google Calendar, Google Docs (for word processing and spreadsheets), YouTube, SlideShare, Amazon, Facebook, twitter and Flickr. Virtually every other Web 2.0 application is a cloud application in this sense.

One of the significant benefits of cloud computing is that it provides scalable access to computing resources and information technology (IT) services. This innovative and accessible resource is an integration of hardware and software which organizations or individuals can utilise anywhere in the world via the internet. Additionally, it will ground the opportunity to penetrate and disseminate the computing resources and information technology (IT) services within the world industries, such as the construction industries. If appropriately implemented and adapted, it will ultimately enhance the productivity, efficiency and effectiveness of the construction industry. The following part of this research will discuss the definitions and concepts of context-aware computing.

\section{Context-Aware \\ Collaboration Tools}

Construction

Information communication technologies (ICT) are reaching their main target of developing an environment in which anyone can easily access any information they may need at any time and from anywhere (cloud computing). On the other hand, the cost reduction of information delivery has drawn the construction industry into an ocean of unmanageable amounts of information (both useful and useless). Therefore, it is important to provide useful information, merely for a specified user, at a given time and according to the user's context (context-awareness). On the other hand, the adaptation of information communication technologies (ICT) and tools in the construction industry is slower compared to other industries. Aziz et al. (2006) asserted that there is limited application of context-aware technology in the construction industry. Furthermore, there are valuable opportunities via evolving the significant enabling technologies of CAID (Context-Aware Information Delivery). Additionally, Aziz et al. (2009) identified some of the enabling technologies for CAID which were classified into location-based services, ubiquitous computing, sensor networks, Radio Frequency Identification (RFID) and profiling technologies.

Awareness of a user's context (such as their role, task, preferences, location and site 
conditions, etc.) in mobile construction applications will enhance the effectiveness of project delivery by providing information and services relevant to a particular context (Fathi et al., 2009). Therefore, awareness of a user's context could provide efficient and effective information, communication and services throughout the entire construction supply chain to enhance the success of construction projects. The following part of this research will briefly discuss the proposed system for integrated, contextaware, cloud computing within the construction industry.

\section{Architectural System of Context-Aware Cloud Computing Information System (CACCIS)}

Beside the characteristics of great fragmentation, similar information is exchanged, processed, manipulated and disseminated throughout the construction industry. Consequently, drawings are redrawn, lists are re-entered, quantities are recounted and materials are ripped out and replaced unnecessarily etc. These examples of unproductive workflows will all be contributors to a decline in productivity. Therefore, the significant objective is to enhance the early coordination and communications along with improving collaboration by bringing the parties within the construction project supply chain together much earlier, in much the same way that design/build brought the architect and contractor together at the project's onset so that any modification one party makes will affect the entire process of the construction project; thus, creating constant communication and efficiencies. Therefore, it is hoped that by proposing the system of context-aware cloud computing (an openly shared collaborative tool), all project stakeholders and supply chain parties, including the design team members, owner, architects, contractors, suppliers, engineers and consultants, will be able to collaborate more accurately and efficiently than in traditional approaches and processes. It is believed that, overall, this system will be more efficient and effective than other systems, models and approaches, such as 3D4D-5D, BIM, Integrated Project Delivery (IPD) and Virtual Design \& Construction (VDC), etc. Implementation of this system will result in enhanced productivity, efficiency and effectiveness within the construction industry. Moreover, the two significant elements of this system, as discussed in previous sections of this research, include context-awareness and cloud computing.

Context-awareness is the awareness of a user's place within the environment context (such as location, roles and responsibilities, identity, preferences, calendar, noise, type of network, time, weather, site conditions, available bandwidth, interests, network type, task, activity, quality of services and network characteristics) which, if acted upon, could enhance the effectiveness of the construction industry by providing the most efficient and valuable information and services relevant to a particular context. On the other hand, the other significant element of this system is cloud computing. As discussed in this research, cloud computing is a technology that uses the internet and central remote servers to maintain data and applications. Furthermore, cloud computing allows consumers and businesses to use applications without installation and access to their personal files on any computer with just an internet access. This technology allows for a much more efficient usage of information technology services over the internet at a dramatically lower cost and with dynamic scalability by centralizing storage, memory, processing and bandwidth. Cloud computing architecture can be divided into two sections: the front end and the back end. They connect to each other through a network. The front end is the side the computer user or client sees. The back end is the cloud section of the system. The ContextAware Cloud Computing Information System (CACCIS) architecture is illustrated in Figure 4 , the front end includes the client's (user's) computer - such as desktops, netbooks, laptops, tablets, mobiles and smartphones - 
along with a web-based application required to access the cloud computing service. It is also known as the cloud computing interface. Services like web-based e-mail and storage programs, such as Gmail, Yahoo Mail, Hotmail and Dropbox are all examples of a computing interface for cloud computing. On the back end of the system, there are various application servers and database (includes data storage) systems beside the server engine (processing server) that together create the 'cloud' of computing services.

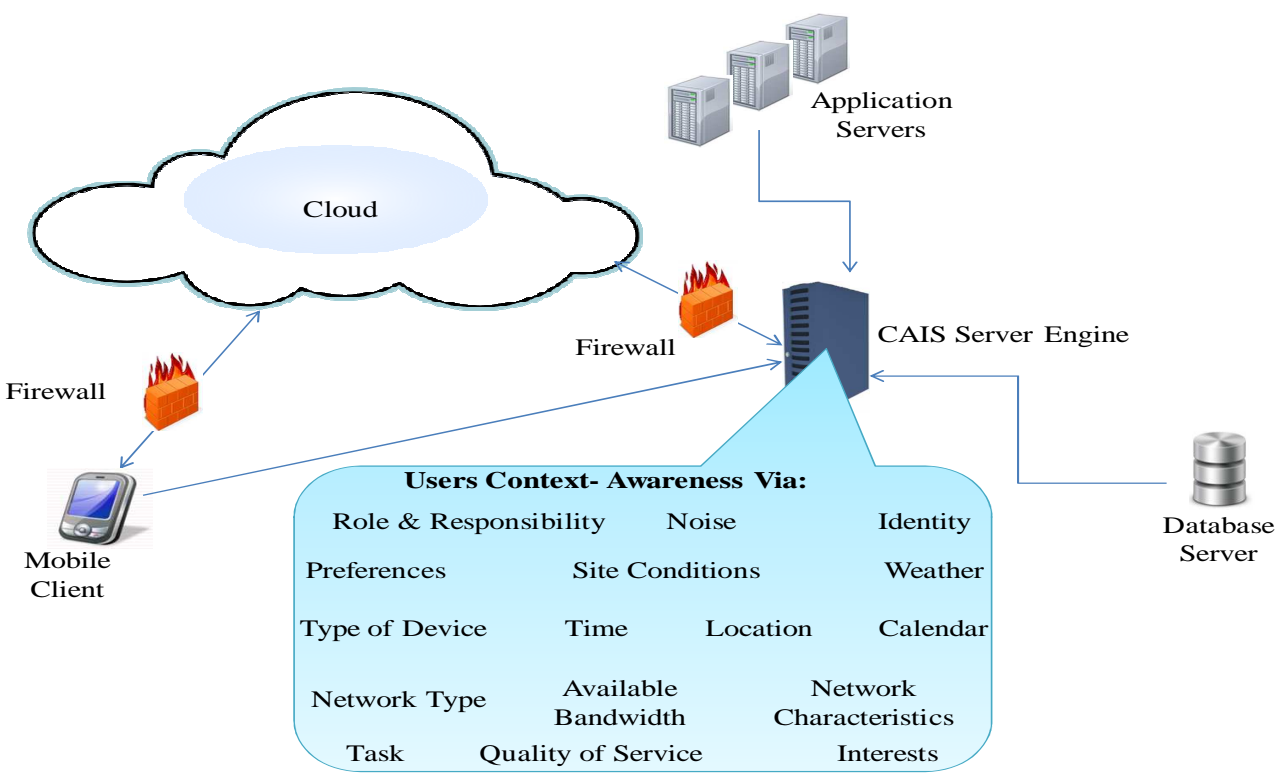

Fig 1: Context-Aware Cloud Computing Information System (CACCIS) Architecture

Fig 1 illustrates that all the data will be received by application servers and database servers which, in turn, will be sending the information to the Context-Aware Information System Server Engine (CAISSE) to process the information. The CAISSE will then send back the relevant information to the users based on selected contexts. Basically, the CACCIS architecture consists of four main components:

(1) The Mobile Client: A mobile device (smartphones and mobile computers) which is able to transmit data and information to the CAIS server engine through the cloud. Also, the client will receive the information from the cloud;

(2) The Firewall: In this system, two firewalls have been proposed of which the first is between the CAIS server engine and the cloud and the second between the cloud and the mobile client. It should be noted that these firewalls are for the security of the data and information which will be transmitted and received by the devices;

(3) CAIS Server Engine: The data which is received from application servers, database servers and mobile clients will be processed by the CAIS server engine. In this phase, many features of the users based on the context defined, such as location, roles and responsibility, identity, preferences, calendar, noise, type of network, time, weather, site conditions, available bandwidth, interests, network type, task, activity, quality of services and network characteristics (user context-awareness) will be selected; and 
(4) The Cloud Server: All the user information which is generated through the CAIS server engine will be transferred through the cloud with authorization through the firewall. Furthermore, this information will then be manipulated and disseminated to the mobile client through the cloud. There are three categories of cloud computing: Infrastructure as a Service (IaaS), Platform as a Service (PaaS) and Software as a Service (SaaS).

Having context-aware applications (such as the location and activity context) allows the application to present information and services that are relevant to the construction supply chain parties. The context-aware application supplies information and services based on the construction supply chain parties' current location by using the Global Positioning System (GPS) for the outdoor location coordinates and a Wireless Local Area Network (WLAN) for the indoor location coordinates. The location coordinates (latitude, longitude and altitude) are then transmitted from the mobile client (mobile client with GPS and WLAN capabilities) to the CAIS Server Engine (CAISSE) through the internet (including the wireless network: WPAN, WLAN, WMAN and WWAN).

According to Omar et al. (2009), although a variety of mobile and context-aware applications already exist, there are still major shortcomings for the potential users. Most of the developed systems are research prototype demonstrator-based and have only been evaluated in small field trials with a limited scope of usage. The scale-up for a bigger usage is impossible for the two following reasons. Firstly, mobile guides might have restrictive hardware requirements like a specific type of PDA (Personal Digital Assistant) and the availability of GPS (Global Positioning System) functionality or client-side software installations. However, new generations of mobile devices, such as smartphones and tablets having larger display sizes, more standardised browsing capabilities and higher speed broadband data transfer, will ease these hardware requirements. Secondly, the availability of extensive and accurate resource data is another limitation. Moreover, due to rapid deployment of wireless communication technologies, such as Wireless Personal Area Network (WPAN), Wireless Local Area Network (WLAN), Wireless Metropolitan Area Network (WMAN) and Wireless Wide Area Network (WWAN), the opportunities for construction industry organizations to offer services to construction supply chain parties and construction project stakeholders within the construction industry along with portable wireless devices (i.e. tablets, laptops, smart phones, netbook computer and etc) are growing. Therefore, to mitigate and overcome the adverse consequences of the issues mentioned, this research proposes the Context-Aware Cloud Computing Information System (CACCIS) as the most valuable construction collaboration tool that will ultimately drive the construction industry to achieve efficient and effective competitive advantages. The last part of this research will provide a brief overview of the significant points discussed within this research and the conclusions.

\section{Conclusion}

The nature of the construction industry is unique among other major industries. Although this industry is mostly one of the early adopters compared to other industries, it is usually slow to adopt the current collaborative tools and technologies. Furthermore, the construction industry is highly project-based and consists of many players with different organizational cultures and objectives. Therefore, in order to achieve successfully the project goals in a timely, effective and efficient manner, the selection and implementation of the appropriate construction collaboration tools for the construction supply chain parties and project stakeholders are vital. On the other hand, the expanding complexity of construction processes and the supply chain necessitates 
the exchanging of increasing amounts of data and information during the construction process.

Overall, this paper has reviewed valuable collaborative tools and technologies as a subset of information and communication technology (ICT) which provides the ability to communicate and exchange data and information easily, efficiently, effectively and accurately at any time or place that might provide major benefits for the construction industry. The collaborative tools and technologies which are discussed in this paper include cloud computing and contextaware computing. Currently, the advances in affordable mobile devices such as smartphones and tablets, improvements in wireless network transfer speeds and enhancements of mobile application performance have facilitated the cloud computing technology's powerful potential to enhance information management within the construction industry. As a result, contextawareness could be integrated with cloud computing in order to ensure the delivery of applicable and reliable information to enhance the collaboration of the parties within the construction supply chain and project stakeholders.

Consequently, this study has set out to introduce and develop the concepts of potentially innovative collaborative tools such as the Context-Aware Cloud Computing Information System (CACCIS) for facilitating the construction supply chain processes and networks to enhance the opportunities for achieving better competitive advantages for the construction industry. Finally, the study presented in this paper is a preliminary survey and is a part of an ongoing research, which will eventually attempt to further enhance the practices and implementation of cloud computing as one of the significant innovative construction collaboration tools.

\section{Acknowledgement}

The authors would like to thank UTM Razak School of Engineering \& Advanced
Technology, Universiti Teknologi Malaysia for sponsoring this research through Research Grant no: 4B012.

\section{References}

Acar, E., Kocak I., Sey, Y. \& Arditi, D. (2005). "Use of Information and Communication Technologies by Small and Mediumsized Enterprises (SMEs) in Building Construction," Construction Management and Economics, 23 (7), 713-722.

Anumba, C. J., Pan, J., Issa, R. R. \& Mutis, I. (2008). "Collaborative Project Information Management in a Semantic Web Environment. Engineering," Construction and Architectural Management, 15 (1), 78-94.

Armbrust, M., Fox, A., Griffith, R., Joseph, A. D., Katz, R. H., Konwinski, A., Lee, G., Patterson, D. A., Rabkin, A., Stoica, I. \& Zaharia, M. (2009). "Above the Clouds: A Berkeley View of Cloud Computing," Technical Report UCB/EECS-2009-28, EECS Department, University of California, Berkeley, [Online], [Retrieved May 2, 2012], http://www.eecs.berkeley.edu/Pubs/TechR pts/2009/EECS-2009-28.pdf.

Aziz, Z., Anumba, C. J., Ruikar, D., Carrillo, P. \& Bouchlaghem, D. (2006). "Intelligent Wireless Web Services for Construction: A Review of the Enabling Technologies," Automation in Construction, 15 (2), 113-123.

Aziz, Z., Anumba, C. \& Peña-mora, F. (2009). "A Road-Map to Personalized Context-Aware Services Delivery in Construction," ITcon, Special Issue Next Generation Construction IT: Technology Foresight, Future Studies, Roadmapping, and Scenario Planning, 14, 461-472, [Online], [Retrieved May 2, 2012], http://www.itcon.org/cgibin/works/Show?2009_30.

Barthelmess, P. (2003). "Collaboration and Coordination in Process-Centered Software Development Environments: A Review of the Literature," Information and Software Technology, 45 (13), 911-928. 
Bittman, T. (2009). "Cloud Computing Inquiries at Gartner," [Online], [Retrieved May 2, 2012] http://blogs.gartner.com/thomas_bittman/2 009/10/29/cloud-computing-inquiries-atgartner/.

Cloud Computing. (2010). "Cloud Computing Use Cases," A White Paper Produced by the Cloud Computing Use Case Discussion Group, Version 4.0, [Online], [Retrieved May 2, 2012],

http://opencloudmanifesto.org/Cloud_Comp uting_Use_Cases_Whitepaper-4_0.pdf.

Erdogmus, H. (2009). "Cloud Computing: Does Nirvana Hide Behind the Nebula?," IEEE Software, 26 (2), 4-6.

Fathi, M. S., Anumba, C. J. \& Carrillo, P. (2009). "Real-Time Mobile Information System for Construction Programme Management," Proceedings of the 2nd Construction Industry Research Achievement International Conference (CIRAIC2009), Kuala Lumpur, Malaysia.

Foley, J. (2008). "Private Clouds Take Shape," InformationWeek, August 9, [Online], [Retrieved May 2, 2012], http://www.informationweek.com/news/se rvices/business/209904474.

Garza., J. M. \& Howitt, I. (1998). "Wireless Communication and Computing at the Construction Site," Automation in Construction, 7 (4), 327-347.

Goscinski, A. \& Brock, M. (2010). "Toward Dynamic and Attribute Based Publication, Discovery and Selection for Cloud Computing," Future Generation Computer Systems, 26 (7), 947-970.

Hoover, J. N. \& Martin, R. (2008). 'Demystifying the Cloud,' InformationWeek Research \& Reports, June, 30-37.
Kim, W. (2009). "Cloud Computing: Today and Tomorrow," Journal of Object Technology, 8 (1), 65-72, [Online], [Retrieved May 2, 2012],

http://jot.fm/issues/issue_2009_01/column 4/.

Kondratova, I. (2003). 'Voice and Multimodal Access to AEC Project Information,' The 10th ISPE International Conference on Concurrent Engineering: The Vision for Future Generations in Research and Applications. J. Cha et al, (eds), Swets \& Zeitlinger. Lisse, Portugal, July 26-30, 755-760, NRC 45829.

Lang, S. Y. T., Dickinson, J. \& Bucha, R. O. (2002). "Cognitive Factors in Design," Computers in Industry, 48, 89-98.

Leung, S.- W., Mak, S. \& Lee, B. L. P. (2008). "Using a Real-Time Integrated Communication System to Monitor the Progress of Quality of Construction Works," Automation in construction, 17 (6), 749-757.

Liu, L. X., Hu, G., Huang, Z. \& Peng, Y. X. (2012). "White Cloud or Black Cloud: Opportunity and Challenge of Spectrum Sharing on Cloud Computing," Advanced Materials Research, 430-432 (1), 1290-1293.

Mell, P. \& Grance. T. (2011). "The NIST Definition of Cloud Computing," National Institute of Standards and Technology, Special Publication 800-145.

Milosevic, D. Z. (2003). Project Management ToolBox: Tools and Techniques for the Practicing Project Manager, New Jersey: John Wiley \& Sons, 584.

Misra, S. C. \& Mondal, A. (2010). "Identification of a Company's Suitability for the Adoption of Cloud Computing and Modelling its Corresponding Return on Investment," Mathematical and Computer Modelling, 53 (3-4), 504-521. 
O’Brien, W. J., Hurley, M. J., Solis, F. A. M. \& Nguyen, T. (2011). "Cognitive Task Analysis of Superintendent's Work: Case Study and Critique of Supporting Information Technologies," Journal of Information Technology in Construction (ITcon), 16, 529556.

Omar, B. \& Ballal, T. (2009). "Intelligent Wireless Web Services: Context-Aware Computing in Construction-Logistics Supply Chain," ITcon, Special Issue Next Generation Construction IT: Technology Foresight, Future Studies, Roadmapping, and Scenario Planning , 14, 289-308, [Online], [Retrieved May 2, 2012], http://www.itcon.org/cgibin/works/Show?2009_20.

Peansupap, V. \& Walker, D. H. T. (2006). "Information Communication Technology (ICT) Implementation Constraints: A Construction Industry Perspective," Engineering, Construction and Architectural Management, 13 (4), 364-379.

Reeves, D. (2009). "Cloud Computing Transforming IT," Burton Group publication, Utah U.S.A 84047-4169, [Online], [Retrieved May 2, 2012], http://net.educause.edu/ir/library/pdf/ECR 0901.pdf.

Sharif, A. M. (2010). "It's Written in the Cloud: The Hype and Promise of Cloud Computing," Journal of Enterprise Information Management, 23 (2), 131-134.

Sultan, N. (2010). "Cloud Computing for Education: A New Dawn?," International Journal of Information Management, 30 (2), 109-116.

Sultan, N. \& Bunt-Kokhuis, S. V. D. (2012). "Organisational Culture and Cloud Computing: Coping with a Disruptive Innovation," Technology Analysis \& Strategic Management, 24 (2), 167-179.
Thomas, P. Y. (2011). " Cloud Computing: A Potential Paradigm for Practicing the Scholarship of Teaching and Learning," The Electronic Library, 29 (2), 214-224.

Voorsluys, W., Broberg, J. \& Buyya, R. (2011). "Introduction to Cloud Computing," Cloud Computing: Principles and Paradigms, John Wiley and Sons, 1-41.

Zhang, X., Kunjithapatham, A., Jeong, S. \& Gibbs, S. (2011). "Towards an Elastic Application Model for Augmenting the Computing Capabilities of Mobile Devices with Cloud Computing," Mobile Networks and Applications, 16 (3), 270-284.

Zhang, X. \& Liu, Y. (2007). "The Impact of ICT on Supplier-Buyer Relationship in Different Types of Supply Chain," Proceedings of 3rd international conference on wireless communications, Beijing, China, 4689-4692. 